\title{
Effect of dietary Auricularia cornea culture supplementation on growth performance, serum biochemistry profile and meat quality in growing-finishing pigs
}

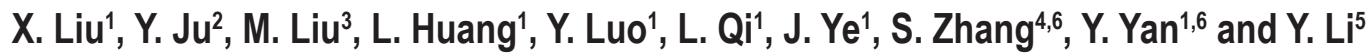 \\ ${ }^{1}$ Guangxi Academy of Agricultural Sciences, Institute of Microbiology, Nanning, 530007, China \\ ${ }^{2}$ Guangxi Academy of Agricultural Sciences, Guangxi Crop Genetic Improvement and Biotechnology Laboratory, \\ Nanning, 530007, China \\ ${ }^{3}$ Lishu Blackland Healthy Food Co., Ltd., Siping, 136599, China \\ ${ }^{4}$ China Agricultural University, State Key Laboratory of Animal Nutrition, Ministry of Agriculture Feed Industry Centre, \\ Beijing, 100193, China \\ ${ }^{5}$ Jilin Agricultural University, Engineering Research Center of Chinese Ministry of Education for Edible and Medicinal Fungi, \\ Institute of Mycology, Changchun 130118, China
}

KEY WORDS: Auricularia cornea culture, growing-finishing pigs, growth performance, meat quality

Received: $\quad 20$ May 2021

Revised: 11 September 2021

Accepted: 15 October 2021

${ }^{6}$ Corresponding author:

e-mail: zhangshuai16@cau.edu.cn; infungi@126.com

$X$. Liu and $Y$. Ju contributed equally to this work.

\begin{abstract}
Auricularia cornea culture (ACC) is a dried product containing Auricularia cornea $(\mathrm{AC})$ mycelium and various metabolites of $\mathrm{AC}$ fermentation. The objective of this study was to investigate the effects of dietary ACC supplementation on growth performance, short-chain fatty acid concentration in faeces, serum biochemical profile and meat quality in growing-finishing pigs. In total, 96 growing pigs with initial body weight $91.94 \pm 7.59 \mathrm{~kg}$, were allotted to one of four dietary treatments for 45 days. Treatments were: basal diet and three experimental diets with $0.3,0.6$ and $1.2 \% \mathrm{ACC}$ addition, respectively. It was shown that pigs fed ACC diets had a greater average daily gain $(P<0.05)$, and also lower glucose content in serum $(P<0.05)$. In comparison with control animals, in pigs fed diets with ACC an increased butyrate content $(P<0.05)$ in faeces and greater monocarboxylate transporter 1 (MCT1) mRNA expression $(P<0.05)$ in the colon were noted. There was also observed an increasing trend concerning a* value $(P=0.09)$ and the higher polyunsaturated fatty acid contents in longissimus dorsi muscle $(P=0.01)$. In conclusion, the dietary ACC addition could improve the growth and health of animals as well as meat quality to a certain degree. So, a 1.2\% ACC supplementation can be recommended for growing-finishing pigs.
\end{abstract}

\section{Introduction}

As a kind of edible and medicinal fungi, Auricularia mushrooms have been widely consumed all around the world. As the fourth most produced mushroom genera, Auricularia has been collected, cultivated and consumed in many countries, such as China, Japan, Vietnam and New Zealand, for hundreds of years (Bandara et al., 2015). Mounting studies have revealed a wide range of pharmacological functions of Auricularia and their derivatives. Auricularia polysaccharides (AP) have been considered to be the major bioactive component, and growing evidences have identified the biological functions of AP with molecular weight (MW) ranging from 4.6 to $3400 \mathrm{kDa}$, including 
antioxidant, immunomodulatory, hepatoprotective and antitumour activities (Miao et al., 2020).

Both crude AP and water-soluble AP were reported to be able to increase the superoxide dismutase (SOD) and glutathione peroxidase (GSH-Px) activities and down-regulate levels of malondialdehyde (MDA) induced by D-galactose in aging mice (Wu et al., 2010; Zhang et al., 2011). Auricularia polysaccharides with MW of less than $110 \mathrm{kDa}$ could stimulate macrophage to secrete interleukin (IL)-1 $\beta$ and IL-6 (Yu et al., 2009). Two kinds of AP (exopolysaccharides) could promote the release of cytokines (IL-6, IL-10 and tumour necrosis factor (TNF)- $\alpha$ ) and nitric oxide (NO) in RAW 264.7 cells line (Zhang et al., 2018). A water-soluble AP extracted by hot water could significantly decrease the levels of low-density lipoprotein cholesterol, triglyceride and total cholesterol in the high-fat diet-induced hyperlipidemic mice (Zeng et al., 2013).

$\beta$-glucan is a sort of functional polysaccharide that widely spreads in the cell wall of bacterial, fungi and cereal seeds (rye, oats and barley, etc.). It possesses various biological functions such as immune function promotion, glucose regulation and anti-infection (Luo et al., 2019). $\beta$-glucan is an important polysaccharide present in mushrooms. Many studies have reported the biological activities of $\beta$-glucan (derived from mushroom), including anti-oxidative, immunomodulatory (Nandi et al., 2014) and anti-inflammatory ones (Ruthes et al., 2013). Most of mushroom $\beta$-glucan was insoluble, with occurrence percentage ranging from 54 to $82 \%$, whereas the percentage of soluble $\beta$-glucan was between 16-46\% (Gern et al., 2008). Several researches indicated that $\beta$-glucan could promote the growth of rats (Belobrajdic et al., 2015) and pigs (Lee et al., 2017), which was concerned with the effects of improving immunity and promoting intestinal health.

Currently, no information regarding the use of Auricularia cornea culture (ACC) and the associated effects on growth and meat quality are available. In this study, we hypothesized that ACC could be utilized as a feed additive that may benefit the growth and health of growing-finishing pigs. Therefore, the objective of this study was to evaluate the effects of dietary ACC inclusion at different levels $(0.3,0.6$ or $1.2 \%)$ on growth performance, serum biochemical profile, faecal short-chain fatty acid (SCFA) contents, carcass characteristics and meat quality in finishing pigs.

\section{Material and methods}

All procedures involving animal handling received the approval of the Institutional Animal Care and Use Committee of Guangxi Academy of Agricultural Sciences (no. GAAS21011201).

Auricularia cornea culture contained a combination of mycelium and fermentation metabolites produced during the specific fermentation process. The mycelium is mainly composed of protein, chitin, cellulose, etc. (Haneef et al., 2017). Firstly, the Auricularia cornea (AC) strain was inoculated into a $1-\mathrm{m}^{3}$ stainless-steel fermentation tank under aseptic conditions. After a 7-day liquid fermentation, the inoculum was used for further solid-state fermentation (SSF). During the 15-day SSF, a specific culture media was inoculated with AC strain and allowed to ferment under sterile, temperature-humidity controlled conditions $\left(26^{\circ} \mathrm{C}\right)$. The entire fermented culture media was subsequently dried at a low temperature $\left(55^{\circ} \mathrm{C}\right)$ to preserve the bioactivity. As AC strain fermented proteins and carbohydrates are present in culture media, they could produce a wide variety of metabolic products, including amino acids, peptides and polysaccharides, such as $\beta$-glucan (Mukhopadhyay and Guha, 2015; Osińska-Jaroszuk et al., 2021), and several undefined metabolites that may have beneficial effects for pigs. The nutrient content of ACC is shown in Table 1.

Table 1. Analysed nutrient content and available energy concentration (MJ/kg) in Auricularia cornea culture (ACC), \%

\begin{tabular}{lr}
\hline Indices & ACC \\
\hline Dry matter & 89.83 \\
Gross energy, MJ/kg & 17.36 \\
Crude protein & 16.37 \\
Ether extract & 4.31 \\
Ash & 4.23 \\
Crude fibre & 6.30 \\
Neutral detergent fibre & 26.54 \\
Acid detergent fibre & 9.42 \\
Total phosphorus & 1.02 \\
Calcium & 0.20 \\
\hline
\end{tabular}

values are the means of six observations, $n=6$

\section{Animals, experimental design and sample collection}

In total, 96 growing-finishing pigs (Duroc $\times$ Landrace $\times$ Yorkshire, $91.94 \pm 7.59 \mathrm{~kg}$ of body weight) were randomly allotted to 4 treatments with 6 replicated pens per treatment (4 pigs per each 
Table 2. Ingredients and calculated nutrient levels of experimental diets, \%, as-fed basis

\begin{tabular}{|c|c|c|c|c|c|c|c|c|}
\hline \multirow{2}{*}{ Indices } & \multicolumn{4}{|c|}{ Grower groups } & \multicolumn{4}{|c|}{ Finisher groups } \\
\hline & Control & $0.3 \%$ ACC & $0.6 \%$ ACC & $1.2 \%$ ACC & Control & $0.3 \%$ ACC & $0.6 \%$ ACC & $1.2 \%$ ACC \\
\hline \multicolumn{9}{|l|}{ Ingredients } \\
\hline maize & 77.61 & 77.59 & 77.59 & 77.59 & 77.91 & 77.91 & 77.91 & 77.87 \\
\hline soybean meal & 15.00 & 15.00 & 15.00 & 15.00 & 13.00 & 13.00 & 13.00 & 13.00 \\
\hline wheat bran & 4.00 & 3.70 & 3.40 & 2.80 & 6.00 & 5.70 & 5.40 & 4.80 \\
\hline soybean oil & 0.70 & 0.72 & 0.72 & 0.72 & 0.80 & 0.80 & 0.80 & 0.82 \\
\hline ACC & 0 & 0.30 & 0.60 & 1.20 & - & 0.30 & 0.60 & 1.20 \\
\hline dicalcium phosphate & 0.60 & 0.60 & 0.60 & 0.62 & 0.42 & 0.42 & 0.42 & 0.42 \\
\hline limestone & 0.90 & 0.90 & 0.90 & 0.88 & 0.84 & 0.84 & 0.84 & 0.84 \\
\hline sodium chloride & 0.35 & 0.35 & 0.35 & 0.35 & 0.35 & 0.35 & 0.35 & 0.35 \\
\hline L-Lys & 0.27 & 0.27 & 0.27 & 0.27 & 0.16 & 0.16 & 0.16 & 0.17 \\
\hline L-Thr & 0.06 & 0.06 & 0.06 & 0.06 & 0.02 & 0.02 & 0.02 & 0.02 \\
\hline L-Trp & 0.01 & 0.01 & 0.01 & 0.01 & - & - & - & 0.01 \\
\hline vitamin-mineral premix ${ }^{1}$ & 0.50 & 0.50 & 0.50 & 0.50 & 0.50 & 0.50 & 0.50 & 0.50 \\
\hline \multicolumn{9}{|l|}{ Analysed nutrient levels } \\
\hline dry matter & 88.31 & 87.92 & 88.59 & 89.24 & 89.45 & 88.89 & 88.20 & 89.07 \\
\hline crude protein & 13.40 & 13.35 & 13.52 & 13.21 & 13.12 & 13.24 & 13.09 & 13.34 \\
\hline gross energy, MJ/kg & 16.24 & 16.05 & 16.39 & 16.50 & 15.91 & 15.87 & 16.19 & 16.22 \\
\hline ash & 3.94 & 4.12 & 4.16 & 4.33 & 4.25 & 4.10 & 4.30 & 4.24 \\
\hline \multicolumn{9}{|l|}{ Calculated nutrient level } \\
\hline $\mathrm{DE}, \mathrm{MJ} / \mathrm{kg}$ & 14.23 & 14.23 & 14.23 & 14.23 & 14.23 & 14.23 & 14.23 & 14.23 \\
\hline SID Lys & 0.73 & 0.73 & 0.73 & 0.73 & 0.61 & 0.61 & 0.61 & 0.61 \\
\hline SID Met & 0.21 & 0.21 & 0.21 & 0.21 & 0.20 & 0.20 & 0.20 & 0.20 \\
\hline SID Thr & 0.46 & 0.46 & 0.46 & 0.46 & 0.40 & 0.40 & 0.40 & 0.40 \\
\hline SID Trp & 0.13 & 0.13 & 0.13 & 0.13 & 0.12 & 0.12 & 0.12 & 0.12 \\
\hline
\end{tabular}

ACC - Auricularia cornea culture, DE - digestible energy, SID - standardized ileal digestible, Lys - lysine, Met - methionine, Thr - threonine, Trp - tryptophan; ${ }^{1}$ provided the following per kg of complete diet: IU: vitamin A 5512 , vitamin $E$ 30, vitamin $D_{3} 2200 ; \mu$ g: vitamin $B_{12} 27.6$, biotin 44; mg: vitamin $\mathrm{K}_{3} 2.2$, D-pantothenic acid 14, pyridoxine 3, riboflavin 4, folic acid 0.7, thiamine 1.5, Mn 40, Cu 100, Fe 75, Zn 75, I 0.35 , Se 0.3

pen), including (1) maize-soybean meal basal diet (control diet); (2) control diet supplemented with $0.3 \%$ ACC; (3) control diet supplemented with $0.6 \%$ ACC; and (4) control diet supplemented with $1.2 \%$ ACC (Table 2). The experiment lasted for 45 days, including 2 phases: the grower phase (days $1-23$, 75-100 kg) and the finisher phase (days 24-45, $100-135 \mathrm{~kg}$ ). During the 45-day feeding period, all pigs were housed in a temperature-controlled room $\left(22-26{ }^{\circ} \mathrm{C}\right)$. Water and feed were available ad libitum. All treatment diets were formulated to meet the nutrient requirements of NRC (2012). Body weight (BW) and feed were weighed at days 0,23 and 45 to determine average daily feed intake (ADFI), average daily gain (ADG) and feed to gain ratio ( $F / G$ ratio). On days 23 and 45, twenty-four fresh faecal samples (one sample per pen) were acquired by rectal palpation for further SCFA analysis (Zhao et al., 2018). The blood samples were collected from pigs with BW close to the average BW in each pen using precaval vein puncture into the 10 -ml vacuette tubes on day 45 ; then the serum was obtained by centrifugation at $3000 \mathrm{r} / \mathrm{s}$ for $15 \mathrm{~min}$ and stored at $-20{ }^{\circ} \mathrm{C}$ until further analysis.
At the end of the trial, a total of 24 pigs (one pig per pen) were selected with BW close to the average BW of each pen. After 18-h fasting, selected pigs were euthanized by exsanguination. About $200 \mathrm{~g}$ of longissimus dorsi muscle (LDM) on the right half of each carcass between the $10^{\text {th }}$ and $12^{\text {th }}$ ribs were acquired for further assessment. The tissue samples from the colon were taken and immediately stored at $-80^{\circ} \mathrm{C}$ until further mRNA expression analysis.

\section{Chemical analysis}

The ACC powder, diets and faeces were analysed for crude protein (CP), dry matter (DM), ash, ether extract (EE), calcium and total phosphorus (AOAC International, 2006). The gross energy (GE) was detected by an Automatic Isoperibol Oxygen Bomb Calorimeter (C2000, IKA, Staufen, Germany). The concentrations of acid detergent fibre (ADF) and neutral detergent fibre (NDF) were measured by a fibre analyser equipment (2010, FOSS, Hillerød, Denmark) (Van Soest et al., 1991).

The SCFA contents were measured via an ion chromatography (883, Metrohm, Herisau, Switzerland) according to the procedure described by 
Wu et al. (2017). Antioxidant parameters, including total antioxidant capacity (T-AOC), superoxide dismutase (SOD) activity, glutathione peroxidase (GSH-Px) activity and malondialdehyde (MDA) content were analysed by assay kits purchased from Jiancheng Bioengineering Ltd. (Nanjing, China). The concentrations of IL-1 $\beta$, IL-2, IL-6, TNF- $\alpha$ were measured using ELISA kits (Jiancheng Bioengineering Ltd., Nanjing, China) with an ELISA Reader (Multiskan, Thermo Fisher Scientific, Waltham, MA, USA). The contents of low-density lipoprotein cholesterol (LDL-C), high-density lipoprotein cholesterol (HDL-C), triglyceride (TG), total cholesterol (TC), glucose (GLU) in serum were analysed by an automatic biochemical analyser (3100, Hitachi, Tokyo, Japan) using corresponding kits (Jiancheng Bioengineering Ltd., Nanjing, China). Fatty acids contents were analysed by gas chromatography (6890, Agilent Technologies, Inc., Santa Clara, CA, USA) according to Sukhija and Palmquist (1988).

\section{Carcass traits and meat quality determination}

After slaughtering, pigs were weighed individually to calculate the hot carcass weight, then were chilled at $4{ }^{\circ} \mathrm{C}$ to measure the carcass length, backfat thickness, loin eye area and marbling. Meat quality assessment, including $\mathrm{pH}$ value, meat colour, cooling loss, shear force and drip loss, were subsequently proceeded.

The dressing percentage was calculated according to the following equation: dressing percentage $(\%)=100 \times$ carcass weight $/$ live body weight. Carcass length was calculated between the $1^{\text {st }}$ rib and the public bone (Latorre et al., 2009). The backfat thickness and loin eye area were determined at the $10^{\text {th }}$ rib according to the procedure described by the Chinese Guidelines on Performance Measurement Technology and Regulations for pig (Ministry of Agriculture of the People's Republic of China, 2014). Three points of the $1^{\text {st }}$ rib, last rib and last lumbar vertebra were recorded to determine the backfat thickness by a vemier caliper. The loin eye area was calculated according to the equation: loin eye area $\left(\mathrm{cm}^{2}\right)=$ loin eye width $(\mathrm{cm}) \times$ loin eye height $(\mathrm{cm}) \times 0.7$. Marbling was evaluated according to the National Pork Producers Council (NPPC) of the United States guidelines (NPPC, 1999).

After making an incision on the LDM, the muscle $\mathrm{pH}$ value was measured using a glass penetration $\mathrm{pH}$ electrode (IS400, SP, AL, USA) at $45 \mathrm{~min}$ post-mortem and recorded as $\mathrm{pH}_{45 \text { min }}$. After storing in a chilling room at $4{ }^{\circ} \mathrm{C}$ for $24 \mathrm{~h}$, the $\mathrm{pH}$ value was measured as $\mathrm{pH}_{24 \mathrm{~h}}$. The meat colour, including $\mathrm{a}^{*}$ (redness), $\mathrm{b}^{*}$ (yellowness) and L* (lightness), was also determined at $45 \mathrm{~min}$ and $24 \mathrm{~h}$ post-mortem using a tristimulus colorimeter (NR, Mingao, Nanjing, China). To determine cooking loss, the steaks were weighed individually in their raw state and then immediately weighed after they had reached their final cooking temperature $\left(80^{\circ} \mathrm{C}\right)$ (Aaslyng et al., 2003). The shear force was measured using a muscle tenderness meter (C-LM3, Mingao, Nanjing, China), after the samples were previously cooked in a water bath at $70{ }^{\circ} \mathrm{C}$ for $20 \mathrm{~min}$ (Ciobanu et al., 2004). The drip loss was measured according to Aaslyng et al. (2003). Briefly, the slice was hung in a plastic bag $\left(4{ }^{\circ} \mathrm{C}\right)$ for $24 \mathrm{~h}$. Then, the drip loss was calculated as: drip loss $(\%)=$ the amount of drip $(\mathrm{g}) /$ initial meat weight $(\mathrm{g})$.

\section{Relative quantification of MCT1 mRNA expression}

Analysis of monocarboxylate transporter 1 (MCT1) mRNA expression was performed as described previously (Metzler-Zebeli et al., 2012; Tudela et al., 2015). The frozen colon sections were pulverized under liquid nitrogen using a pestle and mortar. The total RNA from colonic tissue was extracted using an Invitrogen TRIzol reagent (Thermo Fisher Scientific, Waltham, MA, USA). The RNA quality and quantity were determined on a spectrophotometer (ND-1000, Thermo Fisher Scientific, Waltham, MA, USA). Subsequently, the total RNA was reverse-transcribed into complementary DNA (cDNA) using the Superscript II transcriptase (Invitrogen, Thermo Fisher Scientific, Waltham, MA, USA). Primers for $M C T 1$ were designed based on published sequences (Tudela et al., 2015) and primer information was summarized in Table 3.

Table 3. Primer sequences for Real-Time polymerase chain reaction analysis

\begin{tabular}{lll}
\hline Gene & Primer & Sequence $\left(5^{\prime} \rightarrow 3^{\prime}\right)$ \\
\hline MCT1 & Forward & GGAGACCAGTATAGACGCTGC \\
& Reverse & CTCCTCCTCTTTGGGGCTTC \\
ACTB & Forward & TGCGGGACATCAAGGAGAAGC \\
& Reverse & ACAGCACCGTGTTGGCGTAGAG \\
\hline
\end{tabular}

MCT1 - monocarboxylate transporter $1 ; A C T B$ - $\beta$-actin

The Real-Time polymerase chain reaction (PCR) was performed in a total volume of $10 \mu \mathrm{l}$, which contained $0.2 \mu \mathrm{l}$ each of forward and reverse primers, $1 \mu 1$ cDNA template, $0.2 \mu 1$ ROX Reference Dye and $5 \mu \mathrm{l} \mathrm{SYBR}$ Green mix. Then, the Real-Time quantitative PCR was performed with general cycling 
conditions as follows: pre-denaturation at $95{ }^{\circ} \mathrm{C}$ for $10 \mathrm{~s} ; 40$ cycles of amplification at $95^{\circ} \mathrm{C}$ for $5 \mathrm{~s}$ and $60{ }^{\circ} \mathrm{C}$ for $20 \mathrm{~s}$; melting curve construction from $60{ }^{\circ} \mathrm{C}$ to $99{ }^{\circ} \mathrm{C}$ with a heating rate of $0.1{ }^{\circ} \mathrm{C} / \mathrm{s}$ and fluorescence measurements (Applied Biosystems ${ }^{\mathrm{TM}}$ 7500 Real-Time PCR System; Thermo Fisher Scientific, Waltham, MA, USA).

\section{Statistical analysis}

The data for growth performance, biochemical indices, carcass traits and meat quality were analysed by analysis of variance (ANOVA) using the PROC GLM procedure of SAS (version 9.2, SAS Institute Inc., Cary, NC, USA) followed by Student-NewmanKeuls multiple range tests. The significances were estimated at a probability level of 0.05 .

\section{Results}

As shown in Table 4, there was a trend $(P=0.07)$ concerning final $\mathrm{BW}$ of pigs fed ACC diets. No effects were observed on ADG, ADFI and F/G ratio during days $1-23$. Pigs fed ACC diets showed a higher ADG $(P \leq 0.01)$, whereas ADFI and $\mathrm{F} / \mathrm{G}$ ratio were not influenced during days 24-45 and the overall period.

Table 4. Effects of dietary Auricularia cornea culture (ACC) supplementation on growth performance in growing-finishing pigs

\begin{tabular}{|c|c|c|c|c|c|c|}
\hline \multirow[b]{2}{*}{ Indices } & \multicolumn{4}{|c|}{ Treatments } & \multirow[b]{2}{*}{ SEM } & \multirow[b]{2}{*}{$P$-value } \\
\hline & Control & $\begin{array}{l}0.3 \% \\
\text { ACC }\end{array}$ & $\begin{array}{l}0.6 \% \\
\text { ACC }\end{array}$ & $\begin{array}{l}1.2 \% \\
\text { ACC }\end{array}$ & & \\
\hline IBW & 92.02 & 91.90 & 91.90 & 91.97 & 0.08 & 0.67 \\
\hline FBW & 131.03 & 131.21 & 131.48 & 131.66 & 0.16 & 0.07 \\
\hline \multicolumn{7}{|c|}{ Days 1 to 23} \\
\hline ADG, $g$ & 904 & 906 & 908 & 910 & 4.24 & 0.73 \\
\hline ADFI, $g$ & 2450 & 2392 & 2437 & 2460 & 53.51 & 0.82 \\
\hline$F / G$ & 2.71 & 2.64 & $\quad 2.68$ & 2.70 & 0.06 & 0.84 \\
\hline \multicolumn{7}{|c|}{ Days 24 to 45} \\
\hline$A D G, g$ & $828^{b}$ & $840^{\mathrm{ab}}$ & $849^{a}$ & $853^{a}$ & 4.35 & $<0.01$ \\
\hline ADFI, $g$ & 2525 & 2514 & 2558 & 2578 & 26.07 & 0.32 \\
\hline$F / G$ & 3.05 & 2.99 & 3.01 & 3.02 & 0.03 & 0.69 \\
\hline \multicolumn{7}{|c|}{ Days 1 to 45} \\
\hline$A D G, g$ & $867^{b}$ & $874^{\mathrm{ab}}$ & $880^{a}$ & $882^{a}$ & 3.00 & 0.01 \\
\hline ADFI, $g$ & 2486 & 2452 & 2496 & 2517 & 34.02 & 0.60 \\
\hline$F / G$ & 2.87 & 2.81 & 2.84 & 2.85 & 0.04 & 0.72 \\
\hline
\end{tabular}

IBW - initial body weight, FBW - final body weight, ADG - average daily gain, $A D F I$ - average daily feed intake, F/G - feed to gain ratio, SEM - standard error of the mean; ${ }^{a b}$ - means with different superscripts are significantly different at $P<0.05$

According to obtained data (Table 5), dietary ACC supplementation showed no effects on SCFAs contents on day 23 . Dietary $1.2 \%$ ACC supplementation in diets increased the butyrate content $(P=0.03)$ in pig faeces on day 45 .
Table 5. Effects of dietary Auricularia cornea culture (ACC) supplementation on short-chain fatty acid content measured in fresh pig faeces, $\mathrm{mg} / \mathrm{kg}$

\begin{tabular}{|c|c|c|c|c|c|c|}
\hline \multirow[b]{2}{*}{ Indices } & \multicolumn{4}{|c|}{ Treatments } & \multirow[b]{2}{*}{ SEM } & \multirow[b]{2}{*}{$P$-value } \\
\hline & Control & $\begin{array}{l}0.3 \% \\
\text { ACC }\end{array}$ & $\begin{array}{l}0.6 \% \\
\text { ACC }\end{array}$ & $\begin{array}{l}1.2 \% \\
\text { ACC }\end{array}$ & & \\
\hline \multicolumn{7}{|l|}{ Day 23} \\
\hline acetate & 4256.34 & 4178.04 & 4226.06 & 4469.30 & 222.47 & 0.80 \\
\hline propionate & 3319.74 & 3370.82 & 3507.96 & 3242.03 & 125.71 & 0.52 \\
\hline butyrate & 488.09 & 476.59 & 496.56 & 484.38 & 19.70 & 0.91 \\
\hline total & 8064.16 & 8025.45 & 8230.58 & 8195.72 & 255.92 & 0.93 \\
\hline \multicolumn{7}{|l|}{ Day 45} \\
\hline Scotato & 4895.11 & 4823.64 & 4993.70 & 4924.99 & 108.61 & 0.74 \\
\hline propionate & 3748.29 & 3615.31 & 3691.85 & 3666.96 & 140.13 & 0.92 \\
\hline butyrate & $601.65^{b}$ & $672.18^{\mathrm{ab}}$ & ${ }^{b} 647.25^{a}$ & $706.36^{a}$ & 22.53 & 0.03 \\
\hline total & 9245.05 & 9111.13 & 9332.79 & 9298.31 & 172.54 & 0.81 \\
\hline
\end{tabular}

$\mathrm{n}=6$; SEM - standard error of the mean; ${ }^{\text {ab }}$ - means with different superscripts are significantly different at $P<0.05$

In pigs fed 1.2\% ACC a greater MCT1 mRNA expression $(P=0.01)$ in colon was observed (Figure 1).

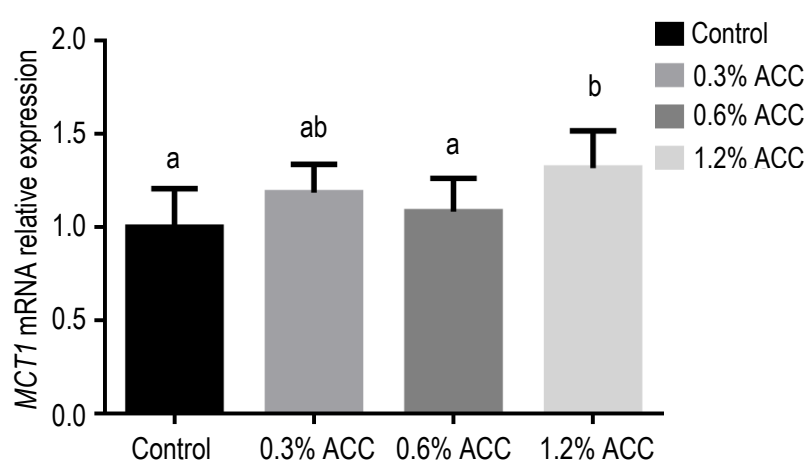

Figure 1. Relative $m R N A$ expression of MCT1 in colon of finishing pigs fed control diet or diets supplemented with $0.3,0.6$ and $1.2 \%$ Auricularia cornea culture (ACC); $n=6 ; M C T 1$ - monocarboxylate transporter 1

As shown in Table 6, in comparison with control animals, pigs fed ACC diets revealed reduced glucose content $(P=0.03)$, and there was no significant effect on GSH-Px, T-AOC, SOD and the other serum indices.

In terms of meat quality (Table 7), there was an increased trend on $\mathrm{a}^{*}$ value $(P=0.09)$. No differences in carcass weight, loin eye area, marbling, shear force, drip loss, and $\mathrm{pH}$ values were observed in pigs fed ACC diets in comparison with control ones.

As shown in Table 8, in pigs fed 0.6 and $1.2 \%$ ACC diets greater linoleic acid $(P=0.02)$ and arachidonic acid $(P<0.01)$ contents were noted in comparison with control ones. Diets supplemented with ACC significantly increase the sums of polyunsaturated fatty acids (PUFA) $(P=0.01)$ and sums of n-6 PUFA $(P<0.01)$ in the LDM. 
Table 6. Effects of dietary Auricularia cornea culture (ACC) supplementation on serum profile of pigs

\begin{tabular}{|c|c|c|c|c|c|c|}
\hline \multirow[b]{2}{*}{ Indices } & \multicolumn{4}{|c|}{ Treatments } & \multirow{2}{*}{\multicolumn{2}{|c|}{ SEM $P$-value }} \\
\hline & Control & $\begin{array}{l}0.3 \% \\
\text { ACC }\end{array}$ & $\begin{array}{l}0.6 \% \\
\text { ACC }\end{array}$ & $\begin{array}{l}1.2 \% \\
\text { ACC }\end{array}$ & & \\
\hline \multicolumn{7}{|l|}{ Day 45} \\
\hline GSH-Px, U/ml & 1109.25 & 1070.67 & 1053.27 & 1122.16 & 41.86 & 0.63 \\
\hline T-AOC, U/ml & 13.43 & 14.66 & 14.72 & 14.94 & 0.80 & 0.55 \\
\hline $\mathrm{SOD}, \mathrm{U} / \mathrm{ml}$ & 75.06 & 82.21 & 79.28 & 80.62 & 4.79 & 0.75 \\
\hline $\mathrm{MDA}, \mathrm{nmol} / \mathrm{ml}$ & 2.65 & 2.39 & 2.76 & 2.58 & 0.24 & 0.73 \\
\hline $\mathrm{HDL}-\mathrm{C}, \mathrm{mmol} / /$ & 1.02 & 1.07 & 1.16 & 1.14 & 0.09 & 0.65 \\
\hline LDL-C, mmol/l & 1.31 & 1.10 & 1.24 & 1.21 & 0.14 & 0.76 \\
\hline $\mathrm{TC}, \mathrm{mmol} / \mathrm{l}$ & 2.49 & 2.67 & 2.41 & 2.52 & 0.19 & 0.80 \\
\hline $\mathrm{TG}, \mathrm{mmol} / \mathrm{l}$ & 0.56 & 0.61 & 0.64 & 0.55 & 0.08 & 0.83 \\
\hline $\mathrm{GLU}, \mathrm{mmol} / \mathrm{l}$ & $2.96^{a}$ & $2.81^{\mathrm{ab}}$ & $2.89^{\mathrm{ab}}$ & $2.69^{b}$ & 0.06 & 0.03 \\
\hline IL-1 $\beta, \mu g / I$ & 27.21 & 27.48 & 26.84 & 26.52 & 1.81 & 0.98 \\
\hline IL-2, ng/l & 488.20 & 476.24 & 493.39 & 496.77 & 12.87 & 0.70 \\
\hline IL-6, $\mu \mathrm{g} / \mathrm{l}$ & 90.94 & 88.70 & 93.22 & 89.38 & 4.69 & 0.91 \\
\hline TNF-a, ng/l & 206.56 & 216.00 & 202.58 & 218.54 & 9.52 & 0.60 \\
\hline
\end{tabular}

$\mathrm{n}=6$; SEM - standard error of the mean; GSH-Px - glutathione peroxidase, T-AOC - total antioxidant capacity, SOD - superoxide dismutase, MDA - malondialdehyde, HDL-C - high-density lipoprotein cholesterol, LDL-C - low-density lipoprotein cholesterol, TC - total cholesterol, TG - triglyceride, GLU - glucose, IL-1 $\beta$ - interleukin- $1 \beta$, IL-2 - interleukin-2, IL-6 - interleukin-6, TNF- $\alpha$ - tumour necrosis factor-a; ${ }^{\text {ab }}$ - means with different superscripts are significantly different at $P<0.05$

Table 7. Effects of dietary Auricularia cornea culture (ACC) supplementation on carcass traits and meat quality of pigs

\begin{tabular}{|c|c|c|c|c|c|c|}
\hline \multirow[b]{2}{*}{ Indices } & \multicolumn{4}{|c|}{ Treatments } & \multirow[b]{2}{*}{ SEM } & \multirow[b]{2}{*}{$P$-value } \\
\hline & Control & $\begin{array}{l}0.3 \% \\
\text { ACC }\end{array}$ & $\begin{array}{l}0.6 \% \\
\text { ACC }\end{array}$ & $\begin{array}{l}1.2 \% \\
\text { ACC }\end{array}$ & & \\
\hline \multicolumn{7}{|l|}{ Carcass traits } \\
\hline carcass length, cm & 106.201 & 105.43 & 3106.08 & 3106.18 & 1.07 & 0.95 \\
\hline hot carcass weight, kg & 98.55 & 97.35 & 599.28 & 398.15 & 1.81 & 0.90 \\
\hline dressing percentage, $\%$ & \% 74.78 & 74.14 & 75.73 & 74.99 & 0.56 & 0.24 \\
\hline loin eye area, $\mathrm{cm}^{2}$ & 42.97 & 43.77 & 46.34 & 46.97 & 2.12 & 0.49 \\
\hline backfat thickness & 14.83 & 14.5 & 14.17 & 14.67 & 0.64 & 0.86 \\
\hline marbling & 1.75 & 1.67 & 1.92 & 2.00 & 0.23 & 0.74 \\
\hline \multicolumn{7}{|l|}{ Meat quality } \\
\hline cooling loss, $\%$ & 21.50 & 21.53 & 22.89 & 21.82 & 0.89 & 0.66 \\
\hline shear force, kg & 33.86 & 32.35 & 33.72 & 32.53 & 1.42 & 0.80 \\
\hline drip loss, $\%$ & 2.25 & 1.54 & 1.76 & 1.93 & 0.2 & 0.12 \\
\hline$L^{*}(45 \min )$ & 35.62 & 36.15 & 37.06 & 37.38 & 0.57 & 0.15 \\
\hline$a^{*}(45 \mathrm{~min})$ & 3.83 & 3.96 & 4.13 & 4.25 & 0.12 & 0.11 \\
\hline$b^{*}(45 \min )$ & 3.55 & 3.76 & 3.46 & 3.52 & 0.17 & 0.62 \\
\hline$L^{*}(24 h)$ & 55.58 & 55.16 & 55.2 & 55.99 & 1.18 & 0.95 \\
\hline$a^{*}(24 h)$ & 10.27 & 10.87 & 11.56 & 11.21 & 0.34 & 0.09 \\
\hline$b^{*}(24 h)$ & 7.51 & 7.36 & 7.82 & 7.92 & 0.37 & 0.69 \\
\hline $\mathrm{pH}_{45 \text { min }}$ & 6.72 & 6.46 & 6.27 & 6.31 & 0.14 & 0.13 \\
\hline $\mathrm{pH}_{24 \mathrm{~h}}$ & 5.91 & 5.87 & 5.78 & 5.82 & 0.09 & 0.74 \\
\hline \multicolumn{7}{|c|}{$\begin{array}{l}\mathrm{pH}_{45 \min }-\mathrm{pH} \text { value } 45 \text { min after post-mortem, } \mathrm{pH}_{24 \mathrm{~h}}-\mathrm{pH} \text { value } 24 \mathrm{~h} \\
\text { after post-mortem, } \mathrm{L}^{*}, \mathrm{a}^{*}, \mathrm{~b}^{*}(45 \mathrm{~min})-\text { meat colour values } 45 \mathrm{~min} \\
\text { after post-mortem, } \mathrm{L}^{*}, \mathrm{a}^{*}, \mathrm{~b}^{*}(24 \mathrm{~h})-\text { meat colour values } 24 \mathrm{~h} \text { after } \\
\text { post-mortem, SEM }- \text { standard error of the mean; values are means } \\
\text { with pooled SEM, } \mathrm{n}=6\end{array}$} \\
\hline
\end{tabular}

Table 8. Effects of dietary Auricularia cornea culture (ACC) supplementation on fatty acids profile in the longissimus dorsi muscle of finishing pigs, $\mathrm{mg} / \mathrm{g}$ of fresh meat

\begin{tabular}{|c|c|c|c|c|c|c|}
\hline \multirow[b]{2}{*}{ Indices } & \multicolumn{4}{|c|}{ Treatments } & \multirow[b]{2}{*}{ SEM } & \multirow[b]{2}{*}{$P$-value } \\
\hline & Control & $\begin{array}{l}0.3 \% \\
\text { ACC }\end{array}$ & $\begin{array}{l}0.6 \% \\
\text { ACC }\end{array}$ & $\begin{array}{l}1.2 \% \\
\text { ACC }\end{array}$ & & \\
\hline \multicolumn{7}{|l|}{ SFA } \\
\hline caproic acid & 0.16 & 0.15 & 0.15 & 0.15 & 0.01 & 0.61 \\
\hline capric acid & 0.16 & 0.16 & 0.15 & 0.15 & 0.01 & 0.37 \\
\hline lauric acid & 0.09 & 0.09 & 0.08 & 0.08 & 0.01 & 0.76 \\
\hline myristic acid & 1.26 & 1.32 & 1.17 & 1.13 & 0.10 & 0.57 \\
\hline palmitic acid & 22.50 & 22.11 & 21.16 & 20.43 & 1.18 & 0.61 \\
\hline heptadecanoic acid & 0.30 & 0.29 & 0.14 & 0.13 & 0.09 & 0.43 \\
\hline stearic acid & 11.44 & 11.14 & 11.10 & 11.58 & 0.95 & 0.98 \\
\hline icosanoic acid & 0.24 & 0.22 & 0.20 & 0.19 & 0.02 & 0.37 \\
\hline heneicosanoic acid & 0.36 & 0.35 & 0.32 & 0.31 & 0.02 & 0.12 \\
\hline \multicolumn{7}{|l|}{ MUFA } \\
\hline palmitoleic acid & 2.59 & 2.64 & 3.18 & 3.07 & 0.19 & 0.10 \\
\hline oleic acid & 48.28 & 47.69 & 46.68 & 47.10 & 2.06 & 0.95 \\
\hline eicosenoic acid & 0.67 & 0.72 & 0.73 & 0.71 & 0.10 & 0.97 \\
\hline \multicolumn{7}{|l|}{ PUFA } \\
\hline linoleic acid & $4.27^{\mathrm{b}}$ & $5.10^{\mathrm{ab}}$ & $5.39^{a}$ & $5.66^{\mathrm{a}}$ & 0.28 & 0.02 \\
\hline a-linolenic acid & 0.33 & 0.31 & 0.34 & 0.35 & 0.06 & 0.98 \\
\hline dihomo-y-linolenic & 0.19 & 0.22 & 0.20 & 0.21 & 0.01 & 0.08 \\
\hline arachidonic acid & $1.21^{\mathrm{b}}$ & $1.39^{\mathrm{ab}}$ & $1.59^{a}$ & $1.62^{\mathrm{a}}$ & 0.07 & $<0.01$ \\
\hline eicosatrienoic acid & 0.10 & 0.09 & 0.10 & 0.09 & 0.01 & 0.90 \\
\hline $\begin{array}{l}\text { eicosapentanoic } \\
\text { acid }\end{array}$ & 0.05 & 0.05 & 0.05 & 0.05 & $<0.01$ & 0.55 \\
\hline docosadienoic acid & 0.04 & 0.04 & 0.05 & 0.04 & $<0.01$ & 0.52 \\
\hline $\begin{array}{l}\text { docosahexaenoic } \\
\text { acid }\end{array}$ & 0.08 & 0.10 & 0.09 & 0.11 & 0.01 & 0.38 \\
\hline$\sum S F A$ & 36.50 & 35.83 & 34.48 & 34.16 & 2.10 & 0.84 \\
\hline$\sum M U F A$ & 51.55 & 51.05 & 50.58 & 50.88 & 2.10 & 0.99 \\
\hline$\sum$ PUFA & $6.26^{b}$ & $7.30^{\mathrm{a}}$ & $7.81^{\mathrm{a}}$ & $8.12^{\mathrm{a}}$ & 0.34 & 0.01 \\
\hline PUFA/SFA & 0.18 & 0.22 & 0.24 & 0.25 & 0.02 & 0.09 \\
\hline$\sum n-6$ PUFA & $5.67^{\mathrm{b}}$ & $6.71^{\mathrm{a}}$ & $7.19^{a}$ & $7.50^{\mathrm{a}}$ & 0.30 & $<0.01$ \\
\hline$\sum n-3$ PUFA & 0.55 & 0.56 & 0.58 & 0.59 & 0.07 & 0.96 \\
\hline$n-6 / n-3$ & 11.04 & 12.83 & 13.21 & 12.89 & 1.16 & 0.56 \\
\hline
\end{tabular}

SEM - standard error of the mean, SFA - saturated fatty acid, MUFA - monounsaturated fatty acid, PUFA - polyunsaturated fatty acid; values are means with pooled SEM, $n=6$; ab - means with different superscripts are significantly different at $P<0.05$

The concentrations of saturated fatty acids (SFA) and monounsaturated fatty acids (MUFA) were not influenced by dietary ACC supplementation. The dihomo- $\gamma$-linolenic content $(P=0.08)$ and PUFA/ SFA ratio value $(P=0.09)$ tended to improve in pigs fed ACC diets.

\section{Discussion}

Polysaccharides are the main component (about $90 \%$ ) of the AC cell wall, and the $\beta$-glucan is the central core of the cell wall (Osińska-Jaroszuk et al., 2021). The $\beta$-glucan concentration in mushrooms ranged from 0.21 to $0.53 \mathrm{~g} / 100 \mathrm{~g}$ (dry weight 
basis) (Rop et al., 2009). It was revealed that the ACC supplementation effectively improve the ADG in pigs. The ameliorated effect might derive from the nutraceutical compounds in ACC. The increased ADG possibly due to the intestinal improvement, as MetzlerZebeli et al. (2012) indicated that dietary $\beta$-glucan could modulate the morphology of piglets and improve intestinal structure. This result is consistent with the previous studies, as Luo et al. (2019) showed that diet supplemented with $\beta$-glucan $(100 \mathrm{mg} / \mathrm{kg}$ ) might significantly increase $\mathrm{ADG}$ and feed conversion ratio, and $0.025 \% \beta$-glucan addition has been shown to augment ADG and ADFI in piglets (Dritz et al., 1995). Li et al. (2006) and Vetvicka et al. (2014) also concluded that supplementing diets with $\beta$-glucan could improve pig health and growth performance.

Pigs fed ACC revealed a higher butyrate level in the current study. The reason was highly due to the $\beta$-glucan proportion, which could act as a unique substrate for the SCFA-generated microbes and modify the relative composition of intestinal microbiota. The mammalian genome does not encode most enzymes required to degrade $\beta$-glucan, and $\beta$-glucan could escape digestion in the foregut and be fermented in the hindgut, resulting in improving SCFA production that altered the microbial ecology in the gastrointestinal tract of pigs. Pieper et al. (2012) reported that feeding $\beta$-glucan enhanced the contents of lactate and propionate in the colon of piglets. Metzler-Zebeli et al. (2012) also showed that oat $\beta$-glucan enhanced caecal and colonic butyrate contents, which could be favourable for intestine development in weaned piglets.

Butyrate is the principal energy source for colonic epithelial cells, which plays a vital role in the epithelial maintenance of intestinal barrier function. The butyrate transportation across the colonocyte luminal membrane is mediated by the MCT1 (Cuff et al., 2002; Plöger et al., 2012). The increased butyrate from the fermented carbohydrates by probiotics has improved MCT1 expression in pigs (Metzler-Zebeli et al., 2012). The carrier-mediated butyrate absorption from the hindgut lumen could be also through MCT1 expression (Tudela et al., 2015). Borthakur et al. (2008) reported that butyrate could activate the MCT1 expression that might occur through a butyrate response element in the MCTI gene promoter region. To further explore the mechanisms involved in the improving effect, we determined the colonic MCT1 mRNA expression, as the generation of butyrate has been positively related to the MCT1 pathway (Cuff et al., 2002). The result showed that 1.2\% ACC supplementation could up-regulate the expression of MCT1 in the colon. The MCT1 mRNA expression and activity were regulated by butyrate, as Cuff et al. (2002) indicated that butyrate presented a concentration- and time-dependent relationship of both MCT1 protein and mRNA. The result is in accordance with the study of Metzler-Zebeli et al. (2012), who showed that dietary $\beta$-glucan could up-regulate MCT1 expression in the caecum. Tudela et al. (2015) investigated the influence of bacterial metabolites on MCT1 expression, and showed that $M C T 1$ expression was higher after incubation with Na-butyrate.

The ACC contains multifarious nutritive materials, especially the polysaccharide from $\mathrm{AC}$, which show various pharmacological activities (Wang et al., 2019). Based on the experimental result, the ACC exhibited a hypoglycemic effect, which was consistent with the study of Wang et al. (2019). In the opinion of Wang et al. (2019), AC appeares to impact glucose metabolism mainly by altering phosphoenolpyruvate carboxykinase (PEPCK) and glucose-6-phosphatase (G-6-Pase) levels in mice's liver. The inhibition of PEPCK and G-6-Pase may effectively regulate blood glucose improvement (Cui et al., 2018).

Meat colour was a vital indicator for the assessment of pork appearance. It was influenced by several factors, such as intramuscular fat content, postmortem glycolysis rate, pigment level and oxidative status of pigment (Van Oeckel et al., 1999). We have revealed that under ACC supplementation $a^{*}$ value had a trend towards increase, which was in accordance with the study in which yeast polysaccharide improved the meat colour (redness had a trend to increase) (Ma et al., 2017). Researches indicated that $\beta$-glucan supplementation could increase $a^{*}$ value of pork and chicken meat (Cho et al., 2013; Luo et al., 2019). The underlying reason for the redder meat colour of $\beta$-glucan-treated pigs might be attributed to an increased mean fibre cross-sectional area or the increased fast twitch muscle fibres (Petersen et al., 1998; Luo et al., 2019).

The fatty acid profile is a valuable parameter of meat quality. The fatty acid profile is the important physical base of meat flavour and has been a primary area of consumer concern (Luo et al., 2019). The present study showed that the proportions of linoleic acid, arachidonic acid, total PUFA and n-6 PUFA in pigs fed $1.2 \%$ ACC diet were increased, in comparison with control animals. Fatty acids are the main components of adipose tissue, the PUFA could only be acquired from diets, as n-3 and n-6 PUFA could not be converted into each other (Liu et al., 2020). To our knowledge, there is limited available literature about dietary AC addition and it's relation to the pork fatty acid profile. The result was 
probably analogical with previous studies that showed two types of fungi, Aspergillus and Saccharomyces, could improve the UFA/SFA ratio and linolenic acid content in broiler meat through its beneficial effects on the intestinal microbiota (Endo and Nakano, 1999; Saleh et al., 2013). Polyunsaturated fatty acids compose more than one unsaturation and are beneficial to health. The n-3 and n-6 PUFAs are necessary for the body and have specific functions, namely anti-inflammatory, vasodilatory and chemotactic effects (Kus-Yamashita et al., 2016). Linoleic acid plays a positive role in reducing blood cholesterol and slowing the development of atherosclerosis (Jandacek, 2017). Arachidonic acid is a fundamental constituent of cell structure and is an essential fatty acid for animals. It impacts the function of specific membrane proteins and plays a vital role in maintaining the integrity of cells and organelle (Tallima and Ridi, 2017). Thus, dietary ACC supplementation could improve PUFA contents in LDM, which is beneficial for human consumption and health. However, the reasons for the changes in the fatty acid composition affected by ACC supplementation are unclear in the current study, so further research is necessary to determine the possible mechanism of such situation.

\section{Conclusions}

The supplementation of Auricularia cornea culture (ACC) to pig diets could improve growth performance, faecal short-chain fatty acid content, polyunsaturated fatty acid contents in longissimus dorsi muscle, and showed a hypoglycemic effect to a certain degree. So, the optimal supplementation of $1.2 \% \mathrm{ACC}$ into growing-finishing pig diets may be recommended.

\section{Acknowledgments}

We also thank Jianchun Su, Deqiang Hou, Yue Shen, and Shuqiang Gao. Special thanks to them for their support in the completion of the current study.

\section{Funding}

This study was financially supported by China Postdoctoral Science Foundation (2021M693806), China Agriculture Research System (CARS20), Postdoctoral Foundation of Guangxi Academy of Agricultural Sciences (GNKB2020038), and the Science and Technology Pioneer of Edible Fungi Industry (GNKM202108).

\section{Conflict of interest}

The authors declared that there is no conflict of interest.

\section{References}

Aaslyng M.D., Bejerholm C., Ertbjerg P., Bertram H.C., Andersen H.J., 2003. Cooking loss and juiciness of pork in relation to raw meat quality and cooking procedure. Food Qual. Prefer. 14, 277-288, https://doi.org/10.1016/S0950-3293(02)00086-1

AOAC International, 2006. Official Methods of Analysis of AOAC International. Current through Revision 1. 18 ${ }^{\text {th }}$ Edition. Gaithersburg, MD (USA)

Bandara A.R., Chen J., Karunarathna S., Hyde K.D., Kakumyan P., 2015. Auricularia thailandica sp. nov. (Auriculariaceae, Auriculariales) a widely distributed species from Southeastern Asia. Phytotaxa 208, 147-156, https://doi.org/10.11646/phytotaxa.208.2.3

Belobrajdic D.P., Jobling S.A., Morell M.K., Taketa S., Bird A.R., 2015. Whole grain barley $\beta$-glucan fermentation does not improve glucose tolerance in rats fed a high-fat diet. Nutr. Res. 35, 162168, https://doi.org/10.1016/j.nutres.2014.12.006

Borthakur A., Saksena S., Gill R.K., Alrefai W.A., Ramaswamy K., Dudeja P.K., 2008. Regulation of monocarboxylate transporter 1 (MCT1) promoter by butyrate in human intestinal epithelial cells: involvement of NF-KB pathway. J. Cell. Biochem. 103, 1452-1463, https://doi.org/10.1002/jcb.21532

Cho J.H., Zhang Z.F., Kim I.H., 2013. Effects of single or combined dietary supplementation of $\beta$-glucan and kefir on growth performance, blood characteristics and meat quality in broilers. $\mathrm{Br}$. Poult. Sci. 54, 216-221, https://doi.org/10.1080/00071668.201 3.777691

Ciobanu D.C., Bastiaansen J.W.M., Lonergan S.M., Thomsen H., Dekkers J.C.M., Plastow G.S., Rothschild M.F., 2004. New alleles in calpastatin gene are associated with meat quality traits in pigs. J. Anim. Sci. 82, 2829-2839, https://doi. org/10.2527/2004.82102829x

Cuff M.A., Lambert D.W., Shirazi-Beechey S.P., 2002. Substrate-induced regulation of the human colonic monocarboxylate transporter, MCT1. J. Physiol. 539, 361-371, https://doi.org/10.1113/ jphysiol.2001.014241

Cui X., Qian D.W., Jiang S., Shang E.X., Zhu Z.H., Duan J.A., 2018. Scutellariae radix and coptidis rhizoma improve glucose and lipid metabolism in T2DM rats via regulation of the metabolic profiling and MAPK/PI3K/Akt signaling pathway. Int. J. Mol. Sci. 19, 3634, https://doi.org/10.3390/ijms19113634

Dritz S.S., Shi J., Kielian T.L., Goodband R.D., Nelssen J.L., Tokach M.D., Chengappa M.M., Smith J.E., Blecha F., 1995. Influence of dietary $\beta$-glucan on growth performance, nonspecific immunity, and resistance to Streptococcus suis infection in weanling pigs. J. Anim. Sci. 73, 3341-3350, https://doi. org/10.2527/1995.73113341x

Endo T., Nakano M., 1999. Influence of a probiotic on productivity, meat components, lipid metabolism, caecal flora and metabolites, and raising environment in broiler production. Anim. Sci. J. 70, 207-218, https://doi.org/10.2508/chikusan.70.207

Gern R.M.M., Wisbeck E., Rampinelli J.R., Ninow J.L., Furlan S.A., 2008. Alternative medium for production of Pleurotus ostreatus biomass and potential antitumor polysaccharides. Bioresour. Technol. 99, 76-82, https://doi.org/10.1016/j.biortech.2006.11.059

Haneef M., Ceseracciu L., Canale C., Bayer I.S., Heredia-Guerrero J.A., Athanassiou A., 2017. Advanced materials from fungal mycelium: fabrication and tuning of physical properties. Sci. Rep. 7, 41292, https://doi.org/10.1038/srep41292 
Jandacek R.J., 2017. Linoleic acid: a nutritional quandary. Healthcare 5(2), 25, https://doi.org/10.3390/healthcare5020025

Kus-Yamashita M.M.M., Filho J.M., Mcdonald B. et al., 2016. Polyunsaturated fatty acids: health impacts. Eur. J. Nutr. Food Saf. 6, 111-131, https://doi.org/10.9734/EJNFS/2016/23018

Latorre M.A., Ripoll G., García-Belenguer E., Ariño L., 2009. The increase of slaughter weight in gilts as a strategy to optimize the production of Spanish high quality dry-cured ham. J. Anim. Sci. 87, 1464-1471, https://doi.org/10.2527/jas.20081362

Lee S.I., Kim J.K., Hancock J.D., Kim I.H., 2017. ß-glucan from mulberry leaves and curcuma can improve growth performance and nutrient digestibility in early weaned pigs. J. Appl. Anim. Res. 45, 209-214, https://doi.org/10.1080/09712119.2016.11 41775

Li J., Li D.F., Xing J.J., Cheng Z.B., Lai C.H., 2006. Effects of $\beta$-glucan extracted from Saccharomyces cerevisiae on growth performance, and immunological and somatotropic responses of pigs challenged with Escherichia coli lipopolysaccharide. J. Anim. Sci. 84, 2374-2381, https://doi.org/10.2527/ jas.2004-541

Liu X.Z., Zhang B., Liu H.S., Zhang J., Liu L., Piao X., Song H., Zhang S., Li Y., 2020. Determination of the available energy values and amino acid digestibility of Flammulina velutipes stem waste and its effects on carcass trait and meat quality fed to growing-finishing pigs. J. Anim. Sci. Biotechnol. 11, 41, https://doi.org/10.1186/s40104-020-00449-y

Luo J.Q., Zeng D.F., Cheng L., Mao X., Yu J., Chen D., 2019. Dietary $\beta$-glucan supplementation improves growth performance, carcass traits and meat quality of finishing pigs. Anim. Nutr. 5 , 380-385, https://doi.org/10.1016/j.aninu.2019.06.006

Metzler-Zebeli B.U., Gänzle M.G., Mosenthin R., Zijlstra R.T., 2012. Oat $\beta$-glucan and dietary calcium and phosphorus differentially modify intestinal expression of proinflammatory cytokines and monocarboxylate transporter 1 and cecal morphology in weaned pigs. J. Nutr. 142, 668-674, https://doi. org/10.3945/jn.111.153007

Ma X., Tian Z., Xiong Y., Qiu Y., Deng D., Wang L., 2017. Effect of yeast polysaccharide on meat quality of finishing pigs. J. Anim. Sci. 95, Suppl. 4, https://doi.org/10.2527/asasann.2017.372

Miao J., Regenstein J.M., Qiu J., Zhang J., Zhang X., Li H., Zhang H., Wang Z., 2020. Isolation, structural characterization and bioactivities of polysaccharides and its derivatives from Auricularia - A review. Int. J. Biol. Macromol. 150, 102-113, https:// doi.org/10.1016/j.ijbiomac.2020.02.054

Ministry of Agriculture of the People's Republic of China, 2014. Technical regulation for testing of carcass traits in lean-type pig (NY/T 825). Beijing (China)

Mukhopadhyay R., Guha A.K., 2015. A comprehensive analysis of the nutritional quality of edible mushroom Pleurotus sajor-caju grown in deproteinized whey medium. LWT - Food Sci. Technol. 61, 339-345, https://doi.org/10.1016/j.Iwt.2014.12.055

Nandi A.K., Samanta S., Maity S., Sen I.K., Khatua S., Devi K.S.P., Acharya K., Maiti T.K., Islam S.S., 2014. Antioxidant and immunostimulant $\beta$-glucan from edible mushroom Russula albonigra (Krombh.) Fr. Carbohydr. Polym. 99, 774-782, https://doi.org/10.1016/j.carbpol.2013.09.016

NPPC (National Pork Producers Council), 1999. Color and Marbling Standards. In: Composition and Quality Assessment Procedures. Des Moines, IA (USA)

NRC (National Research Council), 2012. Nutrient Requirements of Swine: Eleventh Revised Edition. The National Academies Press. Washington, DC (USA), https://doi. org/10.17226/13298
Osińska-Jaroszuk M., Sulej J., Jaszek M., Jaroszuk-Ściseł J., 2021. Applications of fungal polysaccharides. Encycl. Mycolog. 2, 613-628, https://doi.org/10.1016/B978-0-12-8096338.21092-3

Petersen J.S., Henckel P., Oksbjerg N., Sørensen M.T., 1998. Adaptations in muscle fibre characteristics induced by physical activity in pigs. Anim. Sci. 66, 733-740, https://doi.org/10.1017/ S1357729800009310

Pieper R., Bindelle J., Malik G., Marshall J., Rossnagel B.G., Leterme P., Van Kessel A.G., 2012. Influence of different carbohydrate composition in barley varieties on Salmonella Typhimurium var. Copenhagen colonisation in a "Trojan" challenge model in pigs. Arch. Anim. Nutr. 66, 163-179, https://doi.org/10.108 0/1745039x.2012.676814

Plöger S., Stumpff F., Penner G.B., Schulzke J.D., Gäbel G., Martens H., Shen Z., Günzel D., Aschenbach J.R., 2012. Microbial butyrate and its role for barrier function in the gastrointestinal tract. Ann. N. Y. Acad. Sci. 1258, 52-59, https://doi.org/10.1111/ j.1749-6632.2012.06553.x

Rop O., Mlcek J., Jurikova T., 2009. Beta-glucans in higher fungi and their health effects. Nutr. Rev. 67, 624-631, https://doi. org/10.1111/j.1753-4887.2009.00230.x

Ruthes A.C., Carbonero E.R., Córdova M.M., Baggio C.H., Santos A.R.S., Sassaki G.L., Cipriani T.R., Gorin P.A.J., lacomini M., 2013. Lactarius rufus $(1 \rightarrow 3)$, $(1 \rightarrow 6)-\beta$-D-glucans: Structure, antinociceptive and anti-inflammatory effects. Carbohydr. Polym. 94, 129-136, https://doi.org/10.1016/j.carbpol.2013.01.026

Saleh A.A., Hayashi K., Ohtsuka A., 2013. Synergistic effect of feeding Aspergillus awamori and Saccharomyces cerevisiae on growth performance in broiler chickens; promotion of protein metabolism and modification of fatty acid profile in the muscle. J. Poult. Sci. 50, 242-250, https://doi.org/10.2141/ jpsa.0120153

Sukhija P.S., Palmquist D.L., 1988. Rapid method for determination of total fatty acid content and composition of feedstuffs and feces. J. Agric. Food Chem. 36, 1202-1206, https://doi. org/10.1021/jf00084a019

Tallima H., Ridi R.E., 2017. Arachidonic acid: physiological roles and potential health benefits - a review. J. Adv. Res. 11, 33-41, https://doi.org/10.1016/j.jare.2017.11.004

Tudela C.V., Boudry C., Stumpff F., Aschenbach J.R., Vahjen W., Zentek J., Pieper R., 2015. Down-regulation of monocarboxylate transporter 1 (MCT1) gene expression in the colon of piglets is linked to bacterial protein fermentation and proinflammatory cytokine-mediated signalling. Br. J. Nutr. 113, 610-617, https://doi.org/10.1017/S0007114514004231

Van Oeckel M.J., Warnants N., Boucqué C.V., 1999. Measurement and prediction of pork colour. Meat Sci. 52, 347-354, https:// doi.org/10.1016/S0309-1740(99)00012-1

Van Soest P.J., Robertson J.B., Lewis B.A., 1991. Methods for dietary fiber, neutral detergent fiber, and nonstarch polysaccharides in relation to animal nutrition. J. Dairy Sci. 74, 3583-3597, https://doi.org/10.3168/jds.S0022-0302(91)78551-2

Vetvicka V., Vannucci L., Sima P., 2014. The effects of $\beta$-glucan on pig growth and immunity. Open Biochem. J. 8, 89-93, https://doi. org/10.2174/1874091X01408010089

Wang D., Jiang X., Teng S., Zhang Y., Liu Y., Li X., Li Y., 2019. The antidiabetic and antinephritic activities of Auricularia cornea (an albino mutant strain) via modulation of oxidative stress in the $\mathrm{db} / \mathrm{db}$ mice. Front. Immunol. 10, 1039, https://doi. org/10.3389/fimmu.2019.01039 
Wu Q., Tan Z., Liu H., Gao L., Wu S., Luo J., Zhang W., Zhao T., Yu J., Xu X., 2010. Chemical characterization of Auricularia auricula polysaccharides and its pharmacological effect on heart antioxidant enzyme activities and left ventricular function in aged mice. Int. J. Biol. Macromol. 46, 284-288, https:// doi.org/10.1016/j.jijbiomac.2010.01.016

Wu Y., Pan L., Shang Q.H., Ma X.K., Long S.F., Xu Y.T., Piao X.S., 2017. Effects of isomalto-oligosaccharides as potential prebiotics on performance, immune function and gut microbiota in weaned pigs. Anim. Feed Sci. Technol. 230, 126-135, https:// doi.org/10.1016/j.anifeedsci.2017.05.013

Yu M., Xu X., Qing Y., Luo X., Yang Z., Zheng L., 2009. Isolation of an anti-tumor polysaccharide from Auricularia polytricha (jew's ear) and its effects on macrophage activation. Eur. Food Res. Technol. 228, 477, https://doi.org/10.1007/s00217-008-0955-2

Zeng F., Zhao C., Pang J., Lin Z., Huang Y., Liu B., 2013. Chemical properties of a polysaccharide purified from solid-state fermentation of Auricularia auricular and its biological activity as a hypolipidemic agent. J. Food Sci. 78, H1470-H1475, https://doi.org/10.1111/1750-3841.12226
Zhang H., Wang Z.Y., Zhang Z., Wang X., 2011. Purified Auricularia auricular-judae polysaccharide (AAP I-a) prevents oxidative stress in an aging mouse model. Carbohydr. Polym. 84, 638-648, https://doi.org/10.1016/j.carbpol.2010.12.044

Zhang Y., Zeng Y., Men Y., Zhang J., Liu H., Sun Y., 2018. Structural characterization and immunomodulatory activity of exopolysaccharides from submerged culture of Auricularia auricula-judae. Int. J. Biol. Macromol. 115, 978-984, https://doi. org/10.1016/j.jijbiomac.2018.04.145

Zhao J.B., Liu P., Huang C.F., Liu L., Li E.K., Zhang G., Zhang S., 2018. Effect of wheat bran on apparent total tract digestibility, growth performance, fecal microbiota and their metabolites in growing pigs. Anim. Feed Sci. Technol. 239, 14-26, https:// doi.org/10.1016/j.anifeedsci.2018.02.013 